\title{
A Method of Constructing Response Curve for Panoramic Camera
}

\author{
Quan Wei \\ School of Computer Science and Technology on \\ Changchun University of Science and Technology \\ Jilin Changchun, China \\ quanwei@ cust.edu.cn
}

Wang Ying*

School of Computer Science and Technology on Changchun University of Science and Technology Henan Kaifeng, China 1334772745@qq.com

* Corresponding Author

\author{
Wang Yanchun \\ School of Computer Science and Technology on \\ Changchun University of Science and Technology \\ Heilongjiang, China \\ e-mail: wangyc@cust.edu.cn \\ Wang Xuyang, Zhu Yunrui \\ School of Computer Science and Technology on \\ Changchun University of Science and Technology \\ Jilin Changchun, China \\ 294880557@qq.com,1921931497@qq.com,
}

\begin{abstract}
In order to construct high dynamic range image of high quality, a method of constructing response curve for panoramic camera is presented. This method can automatically obtain a small amount of sampling points covered the whole brightness range space of one picture, through grading the range of the brightness, the difference between the sampling point is limited meanwhile the rate of change. The constructed camera response curve shows that the camera response curve can reflect the characteristics of camera better, so the generated image shows details of both bright and dark regions well. Then this paper gets the luminance information of one scene, which has a very important significance in the light information in the virtual reality. But there are also a lot of work to do, such as devoting to improving the sharpness of the high dynamic range image and the contrast between the regions.
\end{abstract}

Keywords- high dynamic image; response curve; exposure; random sampling point; panoramic camera

\section{INTRODUCTION}

For camera, dynamic range refers to the brightness of the maximum and minimum values in the scene. Conventional digital imaging device such as a digital camera can only capture images of the two orders of magnitude range, in order to involve as much as 10 orders of magnitude range of natural scenes, avoid the "overexposure" and "underexposure" phenomenon, extend the traditional camera limited dynamic range, high dynamic range imaging technology has become a research attracted tremendous attention in recent years.

Nowadays, high dynamic range imaging technology has been widely used. High dynamic range imaging technology cannot only be used in the lighting simulation of the object in real scene and generated by the computer ${ }^{[2]}$ and help rendering of the object generated by the computer, it also can avoid the phenomenon such as the brightness, color differences between the images caused by various factors such as the direction and exposure during the image drawing, modeling, and the processing of image construction. Lighting is quite complicated in real situations, such as direct light from the sky and area light source and indirect illumination from the reflection between objects which are difficult to indicate by the virtual light in computer graphics but the illumination based on image can make it, the core technology is to acquire the information carrier which records real scene illumination, that is the construction of high dynamic range panoramic image.

At present, many construction methods of high dynamic range image have been approached, DebevecMalik algorithm ${ }^{[3]}$ and Nayar ${ }^{[4]}$ algorithm, based on them, many scholars improve the algorithm. For high dynamic range panoramic image, there are mainly three methods ${ }^{[5]}$ : the first is simulation of light and constructed image based on the physical illumination model ${ }^{[6]}$, another way is to take many pictures from different directions of image, and then use the image splicing technology combine them, a good way to get a large coverage area at each shot is to use fisheye lens which can be used to cover all area at least two images, then generate a high dynamic range panoramic image. But these two methods have the drawbacks of the complexity of operation and algorithm. There is another way that is to use a special hardware device directly shoot HDR image ${ }^{[7-9]}$. But the effect of this high dynamic range image is bad.

This paper uses a method that applying a group of images with different amounts of exposures to camera response curve to construct high dynamic range image. But the existing methods obtained a camera response curve through computing are too complex and need a long computing time and have a low efficiency. The author proposes a method of automatic choosing sampling points, which make those sampling points distributed in the whole brightness range space. It is fast and efficient in constructing camera response curve which saves a lot of time and can make a better effect than other algorithm. But there are also a lot of work to do. It is necessary to devote 
to improving the sharpness of the high dynamic range image and the contrast between the regions.

\section{THE CONSTRUCTION OF CAMERA RESPONSE CURVE}

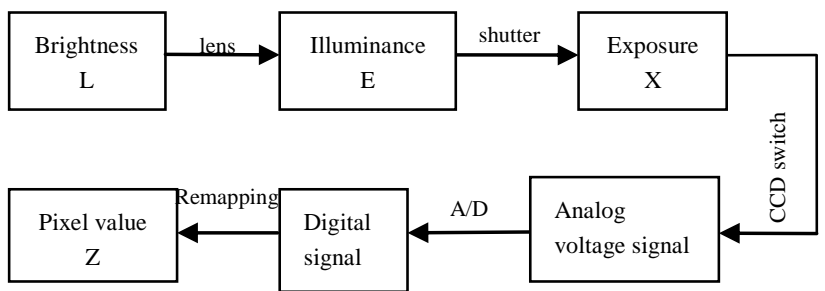

Figure 1. Imaging process of digital camera

Wherever Times is specified, Times Roman or Times New Roman may be used. If neither is available on the word processor, pleas Digital camera imaging process shown in Fig. 1, the procedure is based on the theory of optoelectronics, typically imaged by the CCD. Although the charge output collected by the CCD device is proportional to its illumination, but when these outputs are written on a storage medium, a digital camera will correspond to a non-linear mapping, whose role is to simulate the response characteristics of the film image. The nonlinear mapping constitutes nonlinear relationship between the exposure amount $\mathrm{X}$ and the final pixel value $\mathrm{Z}$. The customized camera response function $\mathrm{f}$ describe the nonlinear relationship between the exposure amount $\mathrm{X}$ and the final pixel value of $\mathrm{Z}$ :

$$
Z=f(X)
$$

Based on the principle of optical imaging system, illuminance $^{[10]}$ value $\mathrm{E}$ received by the camera is proportional to the brightness I of the corresponding points in the scene, which can then be assumed that the characteristic coefficient of camera imaging system is $q$, the effective aperture of the lens is d, the focal length of the lens is f, now the equation can be written as: $E=q I(d / f)^{2}$

The exposure amount can be indicated by Equation (2), where $\Delta t$ is the shutter time. The input parameters when calculating camera response function is a group digital images with different exposure times $\Delta t_{j}$, in order to insure the shooting scene is relatively static, $\Delta t_{j}$ is required to meet a certain threshold $\left(\Delta \mathrm{t}_{\mathrm{j}} \leq \zeta\right)$. Therefore, the equation (2) can be converted into Equation (3). Denote pixel values by $Z_{i j}$ where $\mathrm{i}$ is a spatial index over pixels and $\mathrm{j}$ indexes over exposure times $\Delta t_{j}$. In a certain range, the larger the amount of exposures of the image is, the greater the pixel values is. Now, make a reasonable assumption that $\mathrm{f}$ is monotonely increasing, therefore Equation (3) exists inverse function. Equation (4) can be gotten.

$$
\begin{aligned}
& X=E \Delta t \\
& Z_{i j}=f\left(E_{i} \Delta t_{j}\right) \\
& f^{-1}\left(Z_{i j}\right)=E_{i} \Delta t_{j}
\end{aligned}
$$

Through the Equation (4), its logarithmic Equation (5) can be gotten. Now, define that $g()=\ln f^{-1}()$, then simplify the Equation (5), get Equation (6).

$$
\begin{gathered}
\ln f^{-1}\left(Z_{i j}\right)=\ln E_{i}+\ln \Delta t_{j} \\
g\left(Z_{i j}\right)=\ln E_{i}+\ln \Delta t_{j}
\end{gathered}
$$

Because of the limitation of conventional digital image pixel range, when restoring function $g$ and irradiance $E_{i}$, the range of the corresponding function $g$ is also limited. Making $Z_{\min }$ and $Z_{\max }$ represent the minimum and maximum values of a pixel (integer). It can then be assumed that the number of pixel values is $\mathrm{N}$, the number of pictures is $\mathrm{P}$. Now the needed number of $g\left(Z_{i j}\right)$ is $Z_{\text {max }}-Z_{\text {min }}+1$ and the number of $\ln E_{i}$ is $\mathrm{N}$.Therefore, we can convert the process above to a process of solving least squares objective function:

$$
\begin{aligned}
& \sum_{i=1}^{N} \sum_{j=1}^{P}\left[g\left(Z_{i j}\right)-\ln E_{i}-\ln \Delta t_{j}\right]^{2}=o \\
& \text { Apply } g^{\prime \prime}(z)=g(z-1)-2 g(z)+g(z+1) \quad \text { to the }
\end{aligned}
$$

Equation (7) to smooth the function of $g$.

To make the Equation (7) is to be hyperstaticity, make sure $N(P-1)>\left(Z_{\max }-Z_{\min }\right)$.

\section{THE EFFECT OF THE SAMPLING POINTS FOR THE CAMERA RESPONSE CURVE}

This paper selects 9 sample points in every image. The $\mathrm{x}$-coordinate is defined as pixel value, the $\mathrm{y}$-coordinate is defined as the logarithm of exposure time. Secondly, because the exposure's real value is unknown, assuming the exposure as 1 in order to make the value of $y$ coordinate depend on exposure time. As the relationship between exposure and pixel values is monotonely increasing, then translate the nine sampling points up and down to smooth the curve. Finally, construct camera response curve according to the above-mentioned principle. In addition, the experiments show that the choice of sampling points determines the effect of the curve recovery. Because of the large brightness range of a scene, sampling points prefer to be a smaller change gradient area, then the recovery curve will present the ideal monotone increasing trend. There will be wrong if the sampling points are in a area whose brightness contrast is too large. As shown in Fig. 2, Fig. 2 (1) and (2) are the images of choosing different contrast sampling point, which are taken by a side camera of the panorama camera. Fig. 2 (3) and (4) are the corresponding response curve.
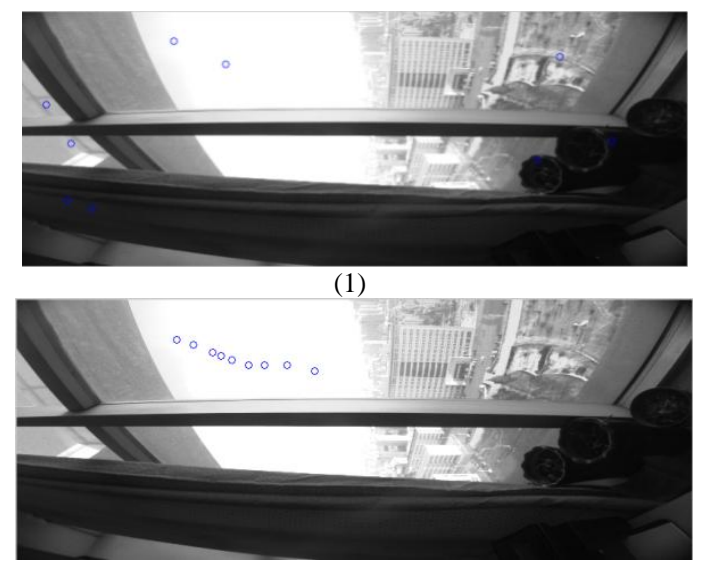
(2)

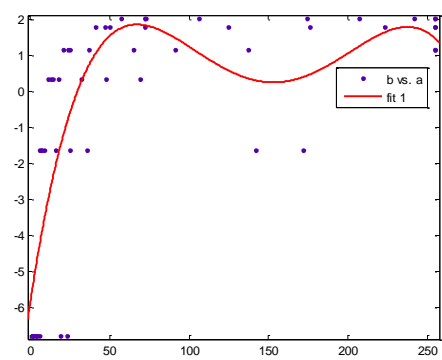

(3)

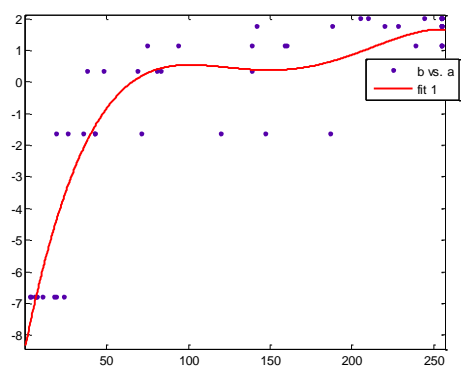

(4)

Figure 2. The choice of sampling points and the constructed result of the curve

\section{A. The method of choosing sampling points for constructing camera response curve}

At present, there are two main methods of obtaining camera response curve:

Firstly, there is no operation about choosing sampling points when computing camera response curve but using all the pixels in the image to fit the camera response curve. Because all the pixels are used, it will increase the time and space complexity of the algorithm.

Secondly, such as its being proposed in the literature 11 销误!未找到引用源。, in the first Chapter 4.61 and the concrete implementation method are as follows:

1) According to the brightness values, the pictures are arranged in descending order.

2) Initializing the unit block list, that is, randomly choose unit block in enough appropriate size and make those unit block stored in the list.

3) The steps of screening the unit block list are as follows:

a) Randomly choose a pixel point from a unit block described above, if the gray value of this pixel in this chosen picture is bigger than the gray value of the other pixel which is in the corresponding point of one picture before the chose picture.

b) Calculate the variance or the standard deviation of the grey value of this unit block, if it exceeds preset threshold, make it remove from the unit block list, or keep it.

4) Repeat Step 3 until all the elements in the unit block complete the test described above.

5) According to the pixels of the unit block screened from the unit block list, the camera response curve can be gotten.
Although the second method can reduce some computation burden and error, it is also highly complex.

\section{B. The method of automatic choosing sampling points for constructing camera response curve}

This paper proposes a method that can automatically obtain a small amount of sampling point covered the whole brightness range space of one picture, through grading the range of the brightness, the difference between the sampling point is limited as well as the rate of change. So the process of the construction of the camera response curve is more rapid and precise.

- Change the exposure time and keep the parameters of the camera constant, $\mathrm{P}$ pictures can be gotten, assuming that Tmin is the minimum exposure value, Tmax is the maximum exposure value. A quick sort is done according to the exposure time of the picture.

- From the $\mathrm{P}$ pictures with different amounts of exposures, choose one picture denoted as PI, then the maximum and the minimum brightness values can be gotten.

- From the maximum and the minimum brightness value, the scope of the brightness can be gotten. The scope can be divided into several grades $R=\lceil N / P\rceil$ so as to the selected sampling points can cover the whole range of the brightness, the span between the points won't be big.

- Randomly select a value $\mathrm{Ri}$ in each grade which is the represent of the brightness in this grade.

- Pick out the coordinates of points whose values are equal to the brightness $\mathrm{Ri}$ which is selected from one range in the picture PI.

- Randomly select one coordinate which acts as the sampling point in this range in the picture PI, denote this coordinate as II

Traverse others pictures in this group in order, select the coordinate II as the first sampling point of this group images, repeat the above operation, then traverse all ranges and then simple points in orders.

\section{THE EXPERIMENTAL RESULTS}

Fig. 3 is the result of sampling points

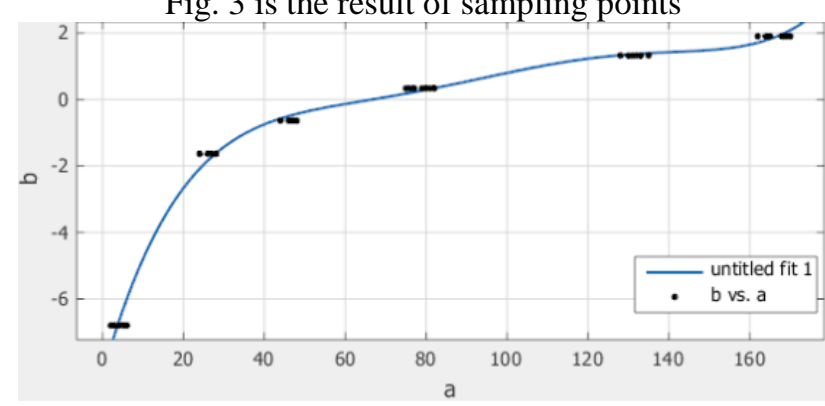

Figure 3. The panoramic camera response curve

Through the camera responsing curve and a group of images with different amounts of exposures, like Figure 4, get he highly dynamic range image Figure 5(1).

Figure $5(2)$ is the result of using matlab. From comparing between Figure 5(2) and Figure 5(1), it can be known that Fig. 5(1) has less noise,more slippery, more subtle color. 

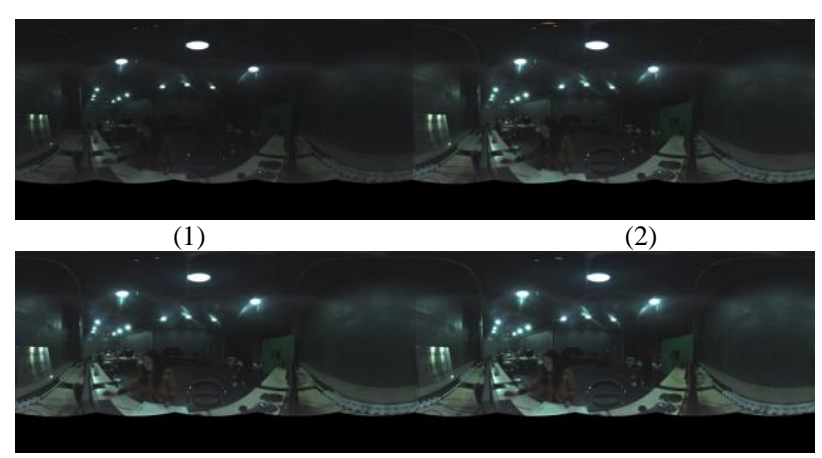

(3)

(4)

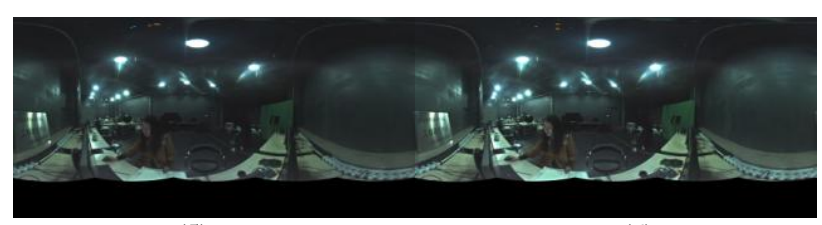

(5)

(6)

Figure 4. a group of images with different amounts of exposures and the construction of hdr dynamic range image

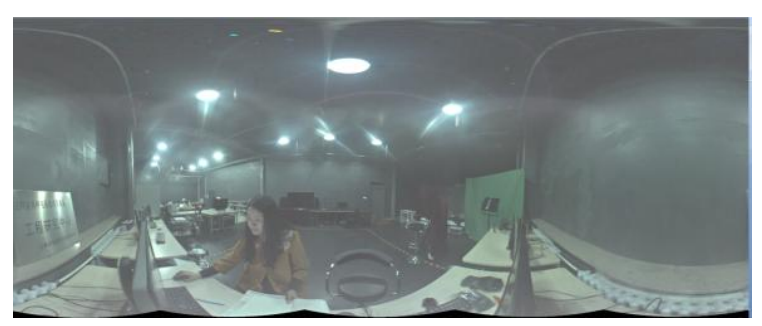

(1)

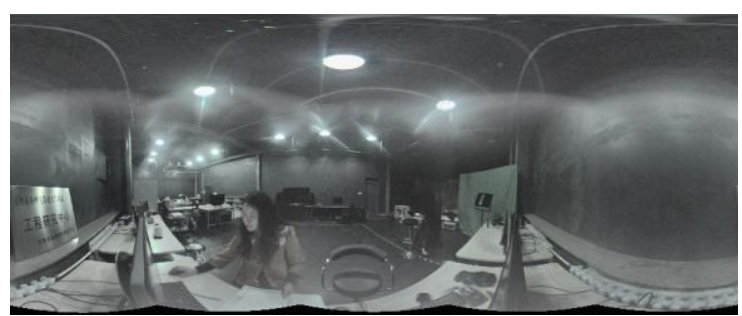

(2)

Figure 5. Differebt HDR image

Because of the limitation of dynamic range, much information of image can be gotten, such as Fig. 4. Because the special light in this laboratory is unable to be shown; but using the method of this paper, the highly dynamic range image shows every detail of laboratory nearly well, such as tables, light. As it is known, the performance of device is limited, in order to construct the image with high dynamic range and show it clearly, tone mapping have been finished to adjust the hue of image, such as Fig. 5(1). Obviously, tone mapping can widen the dynamic range of the image and increase the details of the bright area and dark area in scene, though tone mapping might have an effect upon the hue information of the image, make the contrast decline, and make some details lose. In this paper, the image's dynamic range is $1.8964 \times 10^{6}$ which is close to real scene

\section{ACKNOWLEDGMENT}

Fund Project :Major projects of national science and technology support program(2012BAF12B22), Key scientific and technological projects of Jilin Province(20140204050GX), Major scientific and technological projects of Jilin Province(2012ZDGG004).

First and foremost I would like to show my deepest gratitude to my teacher, Dr. Han Cheng, a respectable, responsible and resourceful scholar, who has provided me with valuable guidance in every stage of the writing of this thesis. Without his enlightening instruction, impressive kindness and patience, I could not have completed my thesis. His keen and vigorous academic observation enlighttens me not only in this thesis but also in my future study.

I shall extend my thanks to Mr. Han for all his kindness and help. I would also like to thank all my teachers who have me to develop the fundamental and essential academic competence.

Last but not least, I'd like to thank all my friends, especially my three lovely roommates, for their encouragement and support.

\section{REFEREBCES}

[1] Zhu Xiuming,Research on Construction and Visualization of High Dynamic Range Image,Zhejiang University,2008.6.3

[2] P.Debevec.Image-based lighting[J].IEEE Computer Graphics and Applications,2002,22(2):26-34.

[3] P.E.Debevec, J.Malik. Recovering High Dynamic Range Radiance Maps from Photographs[C]. in SIGGRAPH97 Conference Proceedings, Annual Conference Series, ACM SIGGRAPH: 1997.369-378.

[4] Shree K. Nayar, Tomoo Mitsunaga. High dynamic range imaging: spatially varying Pixel exposures[C]. New York: in the IEEE Computer Society Conference Proeeedings on Computer Vision and Pattern Recognition, 2000. 472-479.

[5] Zhang Weixiang,Zhou Bingfeng,A Robust HDR Image Calibration Algorithm for Camera Response Function[J],Computer Journal,2006.29(4).658-663.

[6] Larson G. W.,Shakespeare R.. Rendering with Radiance:The Art and Science of Lighting Visualization. San Francisco:Morgan Kaufmann Publishers,1998.

[7] Aggarwal M.,Ahuja N.. Split aperture imaging for high dy-namic range. In:Proceedings of IEEE ICCV, Vancouver, Canada, 2001, II:10-17.

[8] Nayar S. K.,Mitsunaga T.. High dynamic range imaging:Spatially varying pixel exposures. In:Proceedings of IEEE CVPR,Hilton Head Island,South Carolina,2000,472-479.

[9] Mitsunaga T.,Nayar S.K.. Radiometric self calibration. In:Proceedings of IEEE Conference on Computer Vision and Pattern Recognition, Fort Collins, 1999, 374-380.

[10] Debevec P.E.,Malik J.. Recovering high dynamic range radiance maps from photographs. In:Proceedings of the ACMSIGGRAPH97, Los

Angeles,1997,369-378 